\title{
Carpal tunnel syndrome revealing a fibrolipoma of the median nerve
}

\author{
Maroua Slouma $^{1,2}$ (]) Zeineb Zarrouk ${ }^{1,2} \cdot$ Feten Maatoug $^{2,3} \cdot \operatorname{Rim}$ Dhahri ${ }^{1,2} \cdot$ Nada Mansouri $^{2,4} \cdot$ Wajdi Amorri $^{2,3}$. \\ Leila Metoui $^{1,2} \cdot$ Imen Gharsallah $^{1,2} \cdot$ Bassem Louzir $^{2,5}$
}

Received: 2 June 2021 / Revised: 26 June 2021 / Accepted: 28 June 2021 / Published online: 11 July 2021

(c) International League of Associations for Rheumatology (ILAR) 2021

\section{Presentation}

A 25-year-old man presented with a 3-month history of sensory disturbance affecting the three radial digits and the lateral half of the ring finger of his right hand. He reported nocturnal numbness and tingling in the hand's median nerve territory.

He was a military soldier with no medical history.

Physical examination showed elastic soft tissue mass in the volar surface of his right wrist (Fig. 1a). Tinel's sign and Phalen's test were positive. There was no atrophy of the thenar eminence.

Ultrasonography of the right wrist revealed a fusiform enlargement of the median nerve containing parallel hypoechoic bands on longitudinal images and dots on transverse images consistent with nerve fibers separated by hyperechoic substratum consistent with fatty infiltration (Fig. 1b).
Magnetic resonance imaging (MRI) showed a fat-intensity signal fusiform mass that appeared as high signal intensity on T1- and T2-weighted images and low signal intensity on fat-suppressed T2-weighted images surrounding nerve fascicles (Fig. 1c and d). No contrast enhancement was seen after gadolinium administration.

The diagnosis of carpal tunnel syndrome secondary to a fibrolipoma of the median nerve was made based on clinical and imaging features.

The patient had neurolysis and decompression of the median nerve.

Histopathological examination of a perineurium biopsy specimen revealed infiltration of perineurium with mature fibrous and adipose tissue (Fig. 2).

The patient reported a complete resolution of his symptoms and remained asymptomatic after 3 months of treatment.

Maroua Slouma

maroua.slouma@gmail.com

1 Department of Rheumatology, Military Hospital, 1007 Tunis, Tunisia

2 University of Tunis El Manar, Tunis, Tunisia

3 Department of Radiology, Military Hospital, Tunis, Tunisia

4 Department of Pathology, Military Hospital, Tunis, Tunisia

5 Department of Internal Medicine, Military Hospital, Tunis, Tunisia 
Fig. 1 a Presence of a $3 \times 2 \mathrm{~cm}$ fusiform subcutaneous mass over the volar side of the right wrist. b Axial section of volar surface of wrist shows enlargement of the median nerve measuring $17.5 \times 8 \mathrm{~mm}$ (white arrow). No vascular flow was seen within this mass in power Doppler imaging. c T1-weighted sagittal and $\mathbf{d}$ transverse MR images of the wrist showing enlargement of the median nerve (white arrows) with low-weighted signal median nerve fascicles surrounded by bright fat.

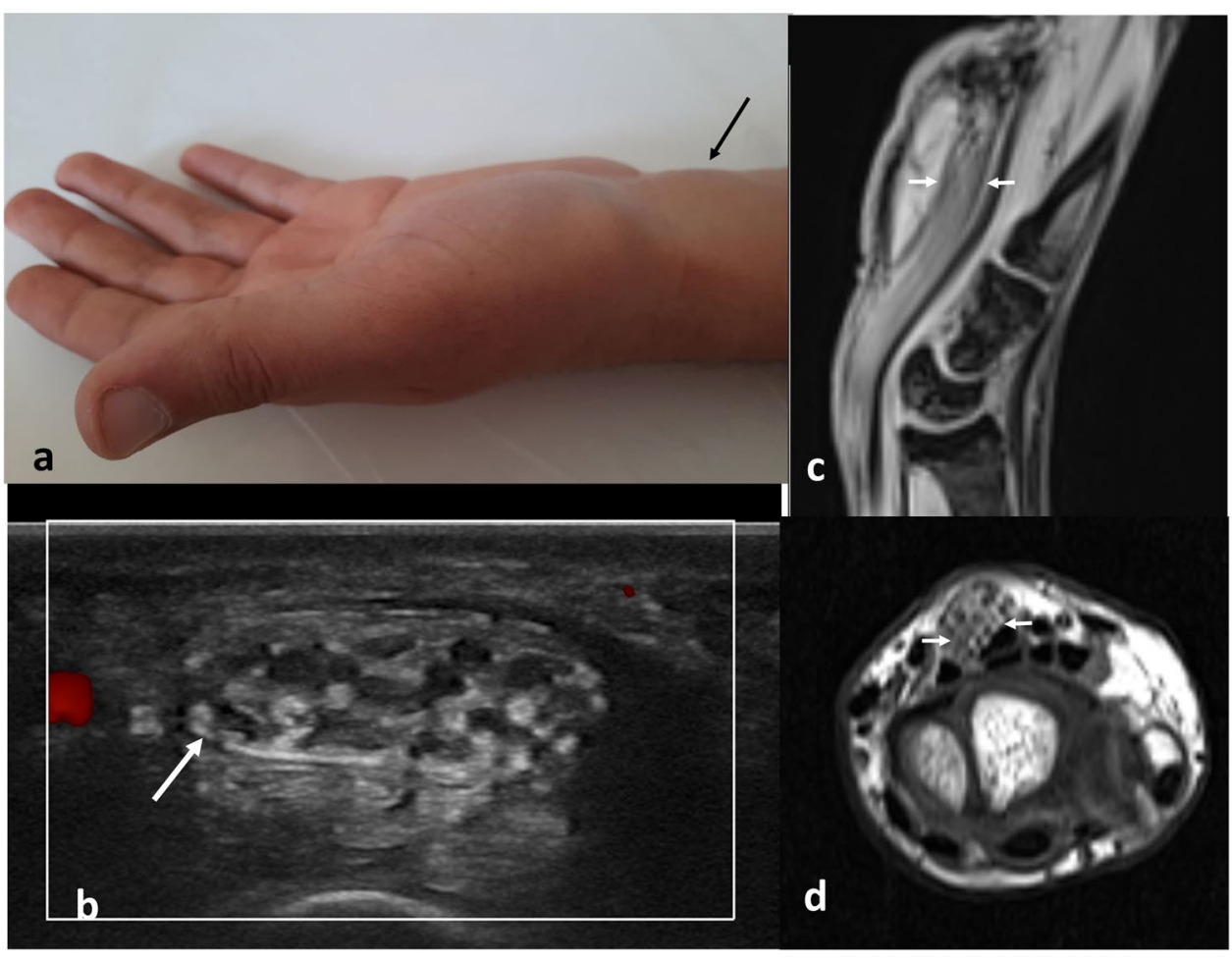

Written informed consent for the case to be published was obtained from the patient.

\section{Discussion}

Fibolipoma of the median nerve is scarce. A literature search in SCOPUS and MEDLINE for English-language sources yielded 35 articles reporting 45 cases of fibrolipoma of the median nerve.

We here reported a new case of fibolipoma of the median nerve revealed by carpal tunnel syndrome.

Fibrolipoma should be considered as a possible etiology of tunnel carpal syndrome, especially in young patients [1,
2]. Clinical manifestations may also include macrodactyly and a mass on the volar surface of the wrist.

Ultrasonography is useful for the diagnosis of rare structural causes of carpal tunnel syndrome [3].

The MRI is the gold standard for the diagnosis of fibrolipoma. It shows a characteristic "cable-like" appearance on axial sections and a "spaghetti-like" appearance on coronal Sects. [4].

The treatment of fibrolipoma is not codified. Several treatments may be suggested, such as neurolysis, epineurotomy, or an excision [5].

Despite its rarity, physicians should be aware of this condition to avoid diagnosis delay and to ensure the appropriate management of these patients. 


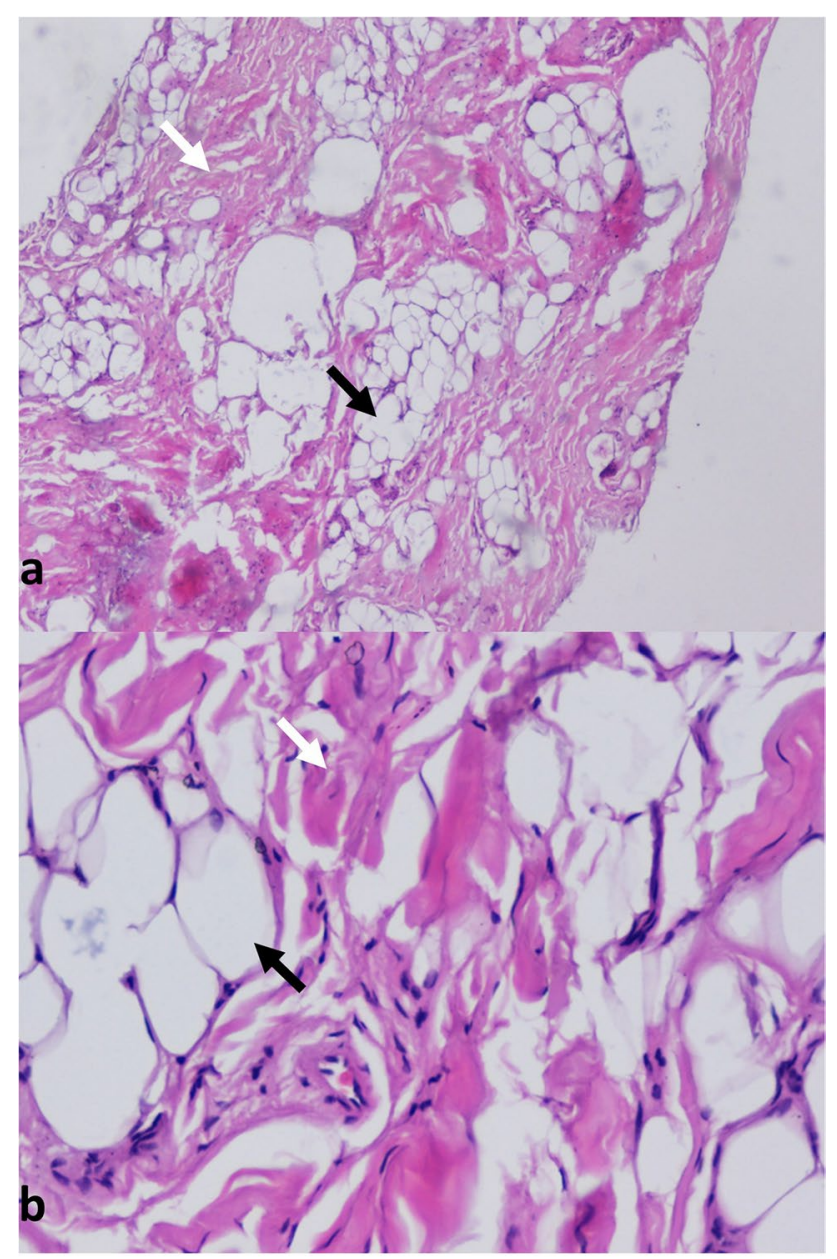

Fig. 2 Histopathological examination of the biopsy specimen showing a mixture of collagen fibers (white arrow) and fatty tissue (black arrow) without intermingling nerve fibers $(\mathrm{HE} \times 200(\mathbf{a})), \mathrm{HE} \times 400$ (b)). $\mathbf{a}=\mathrm{HE} \times 200 . \mathbf{b}=\mathrm{HE} \times 400$
Acknowledgements We would like to thank Dr. Wided LAHMAR for language editing.

\section{Declarations}

Conflict of interest None.

\section{References}

1. Azeemuddin M, Waheed AA, Khan N, Sayani R, Ahmed A (2018) Fibrolipomatous hamartoma of the median nerve with macrodystrophia lipomatosa. Cureus 10(3):e2293. https://doi.org/10.7759/ cureus. 2293

2. Senger JL, Classen D, Bruce G, Kanthan R (2014) Fibrolipomatous hamartoma of the median nerve: A cause of acute bilateral carpal tunnel syndrome in a three-year-old child: A case report and comprehensive literature review. Plast Surg 22(3):201-206

3. Afshar A, Assadzadeh O, Mohammadi A (2015) Ultrasonographic diagnosis of lipofibromatous hamartoma of the median nerve. Iran J Radiol 12(1):e11270. https://doi.org/10.5812/iranjradiol.11270

4. Marom EM, Helms CA (1999) Fibrolipomatous hamartoma: pathognomonic on MR imaging. Skeletal Radiol 28:260-264

5. Marek T, Spinner RJ, Syal A, Wahood W, Mahan MA (2019) Surgical treatment of lipomatosis of nerve: a systematic review. World Neurosurg 128:587-592.e2

Publisher's note Springer Nature remains neutral with regard to jurisdictional claims in published maps and institutional affiliations. 